\title{
Trans-XX-Appliance for Simultaneous eXtrusion and eXpansion
}

\author{
Hampapura Shivananda Dharmesh ${ }^{1}$, Bharathi V Srinivas ${ }^{2}$, Honnalingaiah Kiran ${ }^{3}$, Rajkumar S Alle ${ }^{4}$, Poorna Devadoss ${ }^{5}$
}

\section{Abstract}

Orthodontic disimpaction of impacted maxillary canines requires careful planning of anchorage and proper traction technique to achieve controlled movement of canines through the bone and attached gingiva for maximum esthetics and periodontal health. Trans-XX is a modified transpalatal appliance to simultaneously disimpact an impacted canine along with arch expansion.

Keywords: Canine disimpaction, Maxillary expansion, Transpalatal arch.

Journal of Health Sciences \& Research (2020): 10.5005/jp-journals-10042-1089
\end{abstract}

\section{INTRODUCTION}

Maxillary canine teeth are the second commonly impacted teeth after the third molars, in lieu of long and curvy path of eruption ranging from 2 to $4 \%$ of the population. In $85 \%$ of the patients, the maxillary canines are displaced palatally and $15 \%$ show buccal displacement. Some of the common etiological factors for canine impaction are arch length discrepancy, abnormal position of the dental germ, idiopathic conditions, etc. Reduction of intercanine width can also be an important finding in palatal impaction of canines. ${ }^{1}$ Various methods are used to apply orthodontic traction to disimpact an impacted canine like lasso wires, monkey hooks, pins and cantilever springs, power chain, ligature wires, and double archwires. $^{2}$

\section{Appliance Design}

Transpalatal arch (TPA) was introduced by Robert A Goshgarian in 1972 , and is made of $0.036^{\prime \prime}$ stainless steel wire that spans the palate between the upper first molars.,

Trans-XX is a modification of the TPA to bring about simultaneous extrusion of the both palatally and buccally impacted canines along with slow expansion of the maxillary arch. It is fabricated with $0.031^{\prime \prime}(0.8 \mathrm{~mm})$ stainless steel wire; the outer arm of the modified extended TPA rests against the palatal surfaces of the maxillary posterior teeth. The free ends of the outer arm consist of a helix to engage the E-chain or the ligature wire (Fig. 1).

Slow maxillary expansion is brought about by opening the omega-shaped loop using a three-pronged plier intraorally. For extrusion of canine, the elastic is engaged on to the bracket bonded on the impacted teeth on one end and engaged on to the helix of the free end of the outer arm of the Trans-XX appliance.

The force used to bring about orthodontic canine extrusion is about $0.6 \mathrm{~N}$, while the force generated by the slow expansion is around 2-4 pounds, which are within the physiological limits of the body, can be achieved by this cantilever system appliance. Various other orthodontic systems used for orthodontic extrusion of the maxillary canine like Kilroy spring, elastomeric chain, ligature wire exert about $2.5 \mathrm{~N}$, far beyond the physiological limit of $0.6 \mathrm{~N} .^{1,5}$

\begin{abstract}
${ }^{1-4}$ Department of Orthodontics, RajaRajeswari Dental College and Hospital, Bengaluru, Karnataka, India

${ }^{5}$ Department of Orthodontics, Thai Moogambigai Dental College and Hospital, Chennai, Tamil Nadu, India

Corresponding Author: Bharathi V Srinivas, Department of Orthodontics, RajaRajeswari Dental College and Hospital, Bengaluru, Karnataka, India, Phone: +91 9901402105, e-mail: bharathivs1987@ gmail.com

How to cite this article: Dharmesh HS, Srinivas BV, Kiran H, et al. TransXX-Appliance for Simultaneous eXtrusion and eXpansion. J Health Sci Res 2020;11(1):24-26.
\end{abstract}

Source of support: Nil

Conflict of interest: None

\section{Case Description}

A patient reported with malaligned upper teeth and discomfort during mastication. On extraoral examination, she had straight

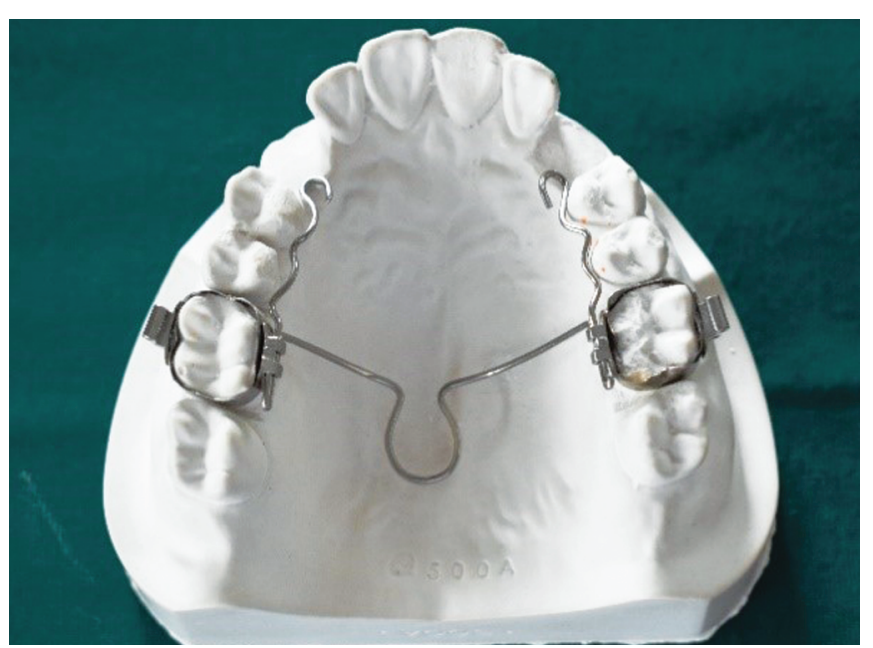

Fig. 1: Trans-XX appliance design fabricated on the cast. Its simple design makes it easier for the application of the orthodontic traction in impacted maxillary canines with unfavorable inclination

(0) The Author(s). 2020 Open Access This article is distributed under the terms of the Creative Commons Attribution 4.0 International License (https://creativecommons. org/licenses/by-nc/4.0/), which permits unrestricted use, distribution, and non-commercial reproduction in any medium, provided you give appropriate credit to the original author(s) and the source, provide a link to the Creative Commons license, and indicate if changes were made. The Creative Commons Public Domain Dedication waiver (http://creativecommons.org/publicdomain/zero/1.0/) applies to the data made available in this article, unless otherwise stated. 

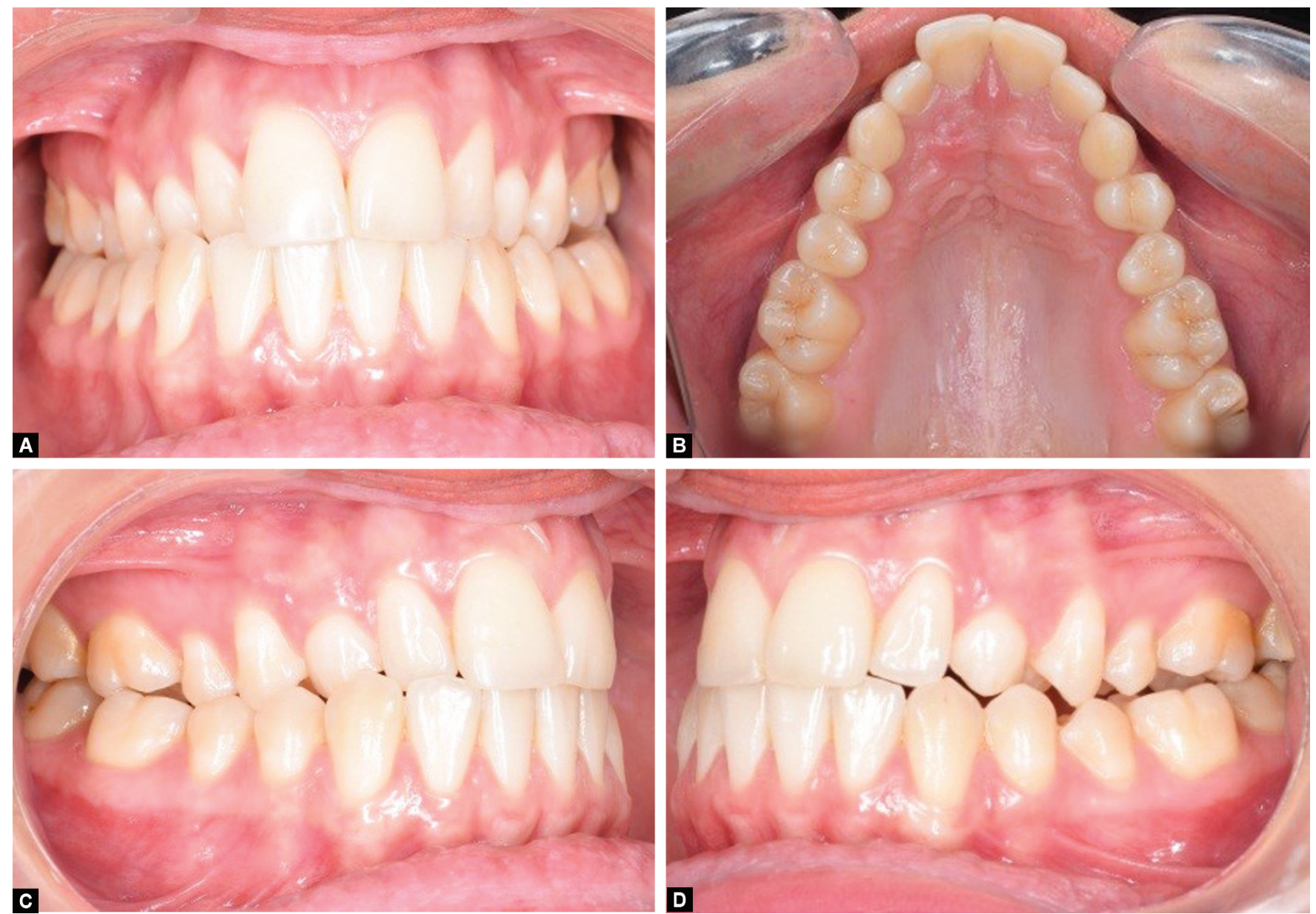

Figs $2 A$ to $D$ : Intraoral photographs

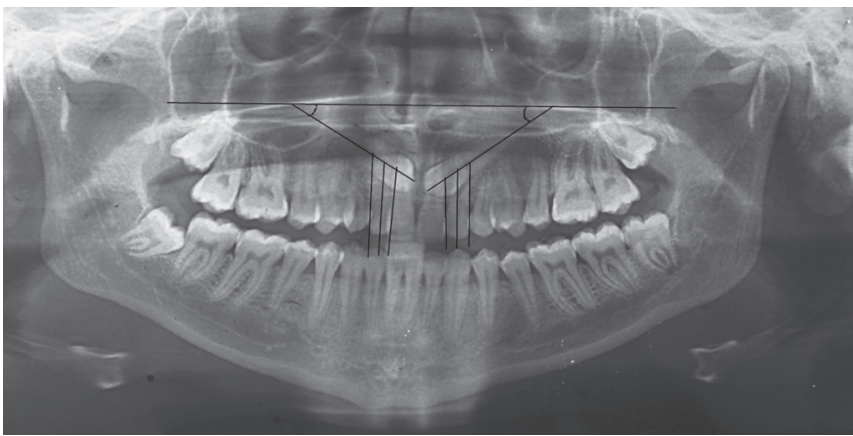

Fig. 3: Radiological findings showing canine cusp tips lying in sector IV

profile, anterior divergent, and having average growth pattern. Intraorally she presented with crowding in both arches, crossbite extending from maxillary lateral incisors till first molar bilaterally, V-shaped upper arch, retained deciduous maxillary canines and impacted permanent canines bilaterally angulated at $42^{\circ}$ and $44^{\circ}$, respectively, both canine cusp tips lying in sector IV according to the classification proposed by Ericson and Kurol ${ }^{6,7}$ (Figs 2 and 3).

The orthodontic treatment plan was to extract the deciduous tooth, surgical exposure, and disimpaction of permanent canines and correction of posterior crossbite, restoring the function and esthetics to the patient. Trans- $X X$ inserted in patient mouth and traction initiated with E-chain (Fig. 4).

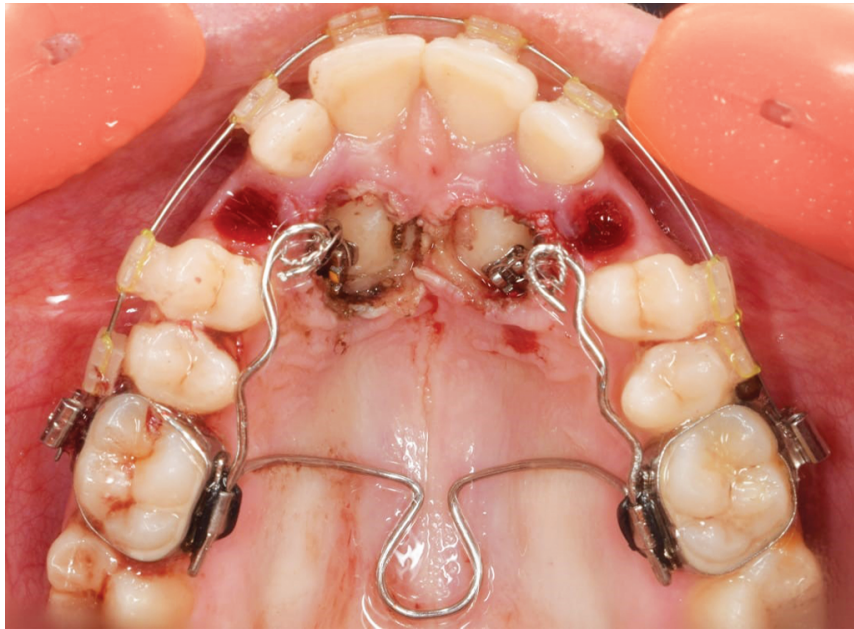

Fig. 4: Trans-XX inserted in patient mouth and traction initiated with E-chain

\section{Conclusion}

Disimpaction of palatally impacted canine is always a challenge to an orthodontist because of its unpredictability. Appliance selection and careful planning of tractional forces on canines are vital for successful treatment results. ${ }^{8}$ The traction of the impacted canines, applying a modified palatal device simultaneously with slow 
maxillary expansion can be effectively and efficiently carried out by Trans-XX appliance. Advantages of this appliance are decreased treatment time and expansion being more physiological because of slow maxillary expansion. Additional clinical procedure and slight discomfort to the patient could be considered as its disadvantages.

\section{References}

1. Potrubacz MI, Chimenti C, Marchione L, et al. Retrospective evaluation of treatment time and efficiency of a predictable cantilever system for orthodontic extrusion of the impacted maxillary canines. Am J Orthod Dentofacial Orthop 2018;154(1):55-64. DOI: 10.1016/j.ajodo.2017.10.027.

2. Goyal B, Munjal S, Singh S, et al. Impacted canine: an arduous taskreview article. J Appl Dent Med Sci 2018;4(1):136-141.

3. Dharmesh HS, Bharathi VS, Kiran H. D-arch: an interdisiplinary approach to absolute anchorage. J Indian Orthod Soc 2019;53:84-86. DOI: 10.4103/jios.jios_229_17.
4. Thomas A, Afshan T, Deru T. Modification of the transpalatal arch for expansion. J Indian Orthod Soc 2017;51(4):289. DOI: 10.4103/03015742.216644.

5. Tepedino M, Chimentia C, Masedua F, et al. Predictable method to deliver physiologic force for extrusion of palatally impacted maxillary canines. Am J Orthod Dentofacial Ortho 2018;153(2):195-203. DOI: 10.1016/j.ajodo.2017.05.035

6. Ciambotti C, Ngan P, Durkee M, et al. A comparison of dental and dentoalveolar changes between rapid palatal expansion and nickeltitanium palatal expansion appliances. Orthod Dentofacial Orthop 2001;119(1):11-20. DOI: 10.1067/mod.2001.110167.

7. Ericson S, Kurol J. Radiographic assessment of canine eruption in children with clinical signs of eruption disturbances. Eur J Ortgod 1986;8(3):133-140. DOI: 10.1093/ejo/8.3.133.

8. Rizvi O, Pattabhiraman V, Nayak A. A modified dis-impaction spring for the impacted canines. APOS Trends Orthod 2015;5(2):83-86. DOI: 10.4103/2321-1407.152063. 